\title{
Clinical characteristics and risk factors of 27 liver failure patients complicated by invasive fungal infections
}

\author{
Longfeng Jiang, Jun Li*, Yaping Han, Yuan Liu, Youde Yan, Nian Chen, Li Dong, \\ Donghui Zhou, Ruiyun Wang
}

Department of Infectious Disease, the First Affiliated Hospital of Nanjing Medical University, Nanjing, China

*Corresponding Author: dr-lijun@vip.sina.com

Received 12 October 2010; revised 28 October 2010; accepted 1 November 2010

\begin{abstract}
To investigate the clinical feature, risk factors and outcome of treatment in patients with liver failure complicated by invasive fungal infections. Retrospective analysis of the clinical data and related factors of 27 patients with liver failure complicated by invasive fungal infections was performed. These patients were admitted from January 2007 to August 2009 in our department. Among them, Candida albicans accounted for 17 cases $(54.84 \%)$, albicans tropicals for 4 cases (12.90\%). Fungal infection in respiratory tract and alimentary tract accounted for $58.06 \%$ and $11 \%$ respectively. $81.25 \%$ of them had fever fluctuating from $37.4^{\circ} \mathrm{C}$ to $40^{\circ} \mathrm{C}$. $81.25 \%$ had elevated white blood cell counts .All had the usage of broad-spectrum of antibiotics, whereas some of them used corticosteroids and had invasive medical manipulation for the treatment. Most patients deteriorated after invasive fungal infections. 21 cases accepted with the treatment of antifungal drugs and mortality rate was $63.00 \%$. It was found that the invasive fungal infection possibility of patients with liver failure significantly increased. To prevent the occurrence of invasive fungal infection, promptly early treatment of liver failure, proper use of antibiotics, cautious use or disuse of corticosteroids, reduction of invasive medical manipulation should be well done. Early detection and treatment of fungal infection are vital to decrease in mortality rate.
\end{abstract}

Keywords: Fungus Diseases; Immunity; Liver Failure; Clinical Feature; Aspergillus

\section{INTRODUCTION}

With the widespread use of broad-spectrum of anti- biotics and invasive medical manipulation for the treatment in clinical, the incidence of invasive fungal infections enhanced and candida albicans and aspergllus were the main infection strains [1]. Due to low immunity, the patients with liver failure are prone to micro-ecological unbalance. As conditional pathogenic bacteria, fungi could increase rapidly. Therefore patients with liver failure can easily merge invasive fungal infections which would lead to high mortality rate. In this study, the clinical data and related factors of 27 patients are retrospectively analyzed, who are treated in our department because of liver failure complicated by invasive fungal infection.

\section{METHODS}

\subsection{Study Object}

27 patients with liver failure complicated by invasive fungal infections were admitted from January 2007 to August 2009 in our department. The clinical data and related factors of them were retrospective analyzed, There are male 19 cases, female 8 cases. Their age ranged from 33 to 82. Etiology: hepatitis B virus infection 16 cases, drug-induced hepatitis 3 cases, hepatitis $\mathrm{C}$ virus infection 1case, autoimmune hepatitis 2 cases, alcoholic hepatitis 2 cases, hepatitis B with hepatocellular carcinoma 3 cases.

\subsection{Diagnostic Criteria}

Diagnostic criteria of liver failure are in accordance with 2006 "liver failure treatment guidelines" [2]. Diagnosis of invasive fungal infection refers to 2003 "Therapeutic Guidelines in Systemic Fungal Infection" and "the diagnosis and treatment guidelines of patients with severe invasive fungal infections" which was developed by Critical Care Medicine Branch of the Chinese Medical Association in 2007 [3,4].

\subsection{Fungi Cultivation and Identification}

The cultivation of fungi was detected using the BAC- 
TEC29210 training apparatus (BD). Identification used fungal identification card (YBC).

\subsection{Record and Analysis}

Records of patients include 1) sex, age, hospitalization days, history of past illness, clinical features and complications; 2) fungal infections: Use of antibiotics and hormones, fungal infection time and type, location , peripheral blood white blood cell count, liver function, invasive operation frequency and so on.

\subsection{Statistical Analysis}

Data are expressed as mean \pm standard error of the mean. Differences between any two groups were determined by t test; $\mathrm{P}<0.05$ was considered statistically significant.

\section{RESULTS}

\subsection{The Fungal Infection Type and Proportion}

There were 31 fungi isolated in 27 patients (Table 1).

\subsection{The Fungal Infection location Distribution}

31 fungi located in various parts, including 18 respiratory tract infection (58.06\%), intestinal infection 11 (35.48\%), fungal blood culture positive 2 (6.45\%) (Table 2).

\subsection{The Clinical Features of Invasive Fungal Infection}

Temperature: 22 cases (81.25\%) showed fever and temperature fluctuated from $37.4^{\circ} \mathrm{C}$ to $40^{\circ} \mathrm{C}$; clinical manifestations: Symptoms of respiratory system infection including throat discomfort, cough, sputum, chest tightness, shortness of breath, etc.; critical cases all had high fever and severe toxemia; principal symptoms of digestive system infection were abdominal distension, abdominal pain and diarrhea; fungal septicemia 2 cases occurred severe high fever and gradually deterioration in constitutional symptom, one of them emerged skin

Table 1. 31 fungal type and proportion.

\begin{tabular}{lll}
\hline type & count & proportion \\
\hline Candida albicans & 17 & $54.84 \%$ \\
Candida tropicalis & 3 & $9.68 \%$ \\
Smooth Candida mycoderma & 1 & $3.23 \%$ \\
Monilia krusei & 5 & $16.13 \%$ \\
Other Candida & 1 & $3.23 \%$ \\
Aspergillus & 4 & $12.90 \%$ \\
\hline
\end{tabular}

Table 2. The fungal infection location distribution.

\begin{tabular}{lll}
\hline location & cases & proportion \\
\hline respiratory tract infection & 18 & $58.06 \%$ \\
intestinal infection & 11 & $35.48 \%$ \\
fungal blood culture positive & 2 & $6.45 \%$ \\
\hline
\end{tabular}

mucous membrane petechia and ecchymosis; Peripheral blood cells: There are 17 cases whose values increased (62.96\%) and neutrophil ratio increased (75\%); liver function: Jaundice deepened 22 cases (81.48\%), prothrombin time prolongation 20 cases $(74.07 \%)$. The white blood cell (WBC), serum bilirubin (TBIL), prothrombin time (PT) were analyzed before and after fungal infection in patient with liver failure. As a result, there was statistical difference (Table 3).

\subsection{Relationship between the Length of Stay in Hospital and the Fungal Infection in Patient}

Among 27 patients, the length of hospitalization time is 5-76 days, average $36 \pm 29$ days, fungal infections occurred 6-55 days after hospitalization, average $18 \pm 13$ days;

Hormone usage: 11 cases used hormone inside or outside hospital, most of them were applied with hydrocortisone $25 \mathrm{mg}-50 \mathrm{mg}$ each time to prevent nosocomial transfusion reaction, yet 2 patients outside the hospital used a large high dose of methylprednisolone. Antibiotics usage: 2 cases administered one type of broad-spectrum antibiotic (7.4\%), more than two types of broad-spectrum antibiotics that included the third generation or fourth generation cephalosporins, quinolones, penicillins and so on. Invasive medical manipulation: abdominal paracentesis 23 case-times, deep venous catheterization 19 case-times, urinary tract intubation 5 case-times, lumbar puncture 1case-time, some patients were operated with two or more than two kinds invasive medical manipulations.

\subsection{Treatment and Outcome}

In addition to positive treatment in primary diseases among 27 liver failure patients complicated by invasive fungal infection, 21 patients were also given fluconazole, itraconazole, voriconazole, caspofungin, allicin and

Table 3. Laboratory index was analyzed ( \pm s)before and after fungal infection.

\begin{tabular}{cccc}
\hline index & before fungal infection & after fungal infection & $\mathrm{P}$ \\
\hline $\mathrm{WBC}\left(10^{\wedge 9 / \mathrm{L})}\right.$ & $4.25 \pm 4.49$ & $9.82 \pm 6.27$ & 0.042 \\
$\mathrm{~TB}(\mathrm{umol} / \mathrm{L})$ & $165.39 \pm 242.50$ & $379.87 \pm 242.29$ & 0.046
\end{tabular}


$\mathrm{PT}(\mathrm{S})$

$22.43 \pm 6.02$

$32.38 \pm 10.08$

0.045

other anti-fungal treatment, and some of them even used combined drug therapy. Result: Among 27 liver failure patients complicated by invasive fungal infection, mortality rate was $63.00 \%$, which included auto-discharged patients. 3 patients suffered from fungal pneumonia and 1 case having fungal sepsis died.

\section{DISCUSSION}

Fungi are widespread in nature as conditional pathogen, which exist in skin or mucous membrane and cause infection by invasion when organism is in low immunity [5]. Because of long time hospitalization, liver failure patients were easy to show various complications such as ascites, water-electrolyte imbalance and so on. Conducted many kinds of invasive medical manipulation which include abdominal paracentesis, deep venous catheterization etc. Long term use of broad-spectrum antibiotics. Due to above factors, the opportunity of invasive fungal infections significantly increases. Liver failure patients would be further aggravate in liver damaged, even lead to death if complicated by infection [6].

The pathogenic fungi of 27 liver failure patients with invasive fungal infection were mainly Candida, which was consistent with the results reported by Zhang $\mathrm{Xu}-$ ehai, etc. [7] and Xie Min et al. [8]. This result illustrated that Candida were widely distributed in nature and stronger pathogenicity in organism. Aspergillus infection was once considered uncommon in the past [9], but aspergillus infection rate in this group was $12.90 \%$ (4/27), all for lung infection, and mortality rate was $75 \%$. The infection sites' constitution ratios were respiratory tract infections (58.06\%), intestinal tract infections (35.48\%). The above summary indicated that spectrum of fungal infection in severe hepatitis patients was expanding with the improvement of diagnosis. The disease severity was more complex and more emphasis should be laid.

Liver failure patients combined with invasive fungal infection were not obvious and specific clinical manifestations. It was not easy to identify the symptoms of liver failure itself. After fungal infections, the majority of patients would be exacerbated. The white blood cell (WBC), serum bilirubin (TBIL), prothrombin time (PT) were analyzed before and after fungal infection in patient with liver failure, there was statistically different .These patients occurred conditions (i.e., unexplained fever, deteriorated disease progress, aggravated systemic symptoms, poor effect of application broad spectrum antibiotics) should be highly vigilant against invasive fungal infection. Routine fungal examination should be carried out to facilitate early diagnosis and give antifungal treatment on time.

In this group, 27 cases with fungal infections occurred in 6-55 days after admission, all existed in broad-spectrum antibiotic usage and invasive operation. Part of them used hormone inside or outside hospital .According to the report by Xie Min et al. [8], application of broad-spectrum antibiotics, invasive operation, hormone usage, and diseases severity etc., were closely related to liver failure complicated by fungal infections.

Because of the complexity of fungal infection, multitiered treatment were advocated in current treatment, including preventive therapy, empirical therapy, preemptive therapy and targeted therapy [4], specific fungus preventive treatment in clinical work for high-risk patients could reduce incidence of invasive fungal infection [10]. Amphotericin B, fluconazole, itraconazole, and voriconazole are main therapeutical drugs for IFI now. Amphotericin B has obvious adverse reaction, and most patients are hard to tolerate. It has been reported that fluconazole had good anti-fungus effect [11]. The antimicrobial spectrum of fluconazole is narrow, and it is mainly sensitive to candida albicans, but not valid for non-candida albicans and aspergillus. Furthermore, candidal drug resistance obviously increases after long-term use [12]. Itraconazole has certain hepatotoxicity, so it is cautiously used in severe hepatitis patients. Voriconazole is a type of broad spectrum antifungal agent, and it has good safety. Antifungus spectrum of voriconazole covers main pathomycete such as candida, aspergillus, Cryptococcus and it is now a good choice for IFI therapy [12]. Therefore, even though most patients were executed anti-fungal treatment, mortality was $63.00 \%$, according to report Xie Min et al. [7]. The mortality of liver failure patients without complicated by fungal infections was $37.78 \%$. This indicated that invasive fungal infection would make liver failure more serious and increase the mortality rate significantly further aggravated the original liver failure and increased the mortality rate significantly in liver failure patients.

Currently the patients with liver failure do not have specific treatment, but only early diagnosis and early treatment; actively to prevent liver cell necrosis and promote liver regeneration, positively treat etiology and prevent complications [14]. For the liver failure patients, according to clinical data analysis of invasive fungal infection in this paper, we believe that some clinical work should be paid attentation to, including 1) treat primary disease, strengthen nutritional support , stabilize internal environment, enhance body resistance; 2) rational use of antibiotics, avoidance of the abuse in broad-spectrum antibiotics and careful use of hormones; 3) reduction of invasive operation as far as possible, strict implementa- 
tion of the aseptic operation, intensive care etc., which will be helpful to decrease the incidence of fungal infection in liver failure patients; 4) for liver failure patients, closely monitor the changes of clinical manifestations in the course of treatment, if situations appear such as the new symptoms or signs unexplained original disease, sudden deterioration of stable condition , invalidity of antibiotic therapy. We should be highly vigilant against the occurrence of fungal infection, retaining appropriate specimens for etiology isolation, identification and acquisition of drug susceptibility results as soon as possible, in order to achieve early diagnosis in fungal infection. Moreover, a number of new rapid detection methods included fungal cell wall compounds and fungal genes DNA etc. were increasingly concerned [15]. Combination of Various diagnostic methods was beneficial to the early diagnosis of invasive fungal, and provided evidence for the early and effective treatment of fungal infections, thus further improving the survival rate of liver failure patients.

\section{ACKNOWLEDGEMENTS}

This study was supported by the National Natural Science Foundation, the funding for Jiangsu Province Key Laboratory and key medical personnel in Infectious Diseases and the funding for Guiding Research Projects of Health Department of Jiangsu Province, China.

\section{REFERENCES}

[1] Zhou, H.H., De, M.T., Guo, Z.L., et al. (2010) Clinical characteristics and therapeutic analysis of invasive fungal infection in chronic severe hepatitis patients. Medical Science, 35, 537-542.

[2] Liver Failure and Artificial Liver Group, Chinese Society of Infectious Diseases, Chinese Medical Association, Severe Liver Diseases and Artificial Liver Group, Chinese Society of Hepatology, Chinese Medical Association (2006) Diagnostic and treatment guidelines for liver failure. Journal of Clinical Hepatology, 9, 321-324.

[3] Richardson, I.M.D. and Jones, B.L. (2003) Therapeutic guidelines in systemic fungal infections. 3rd Edition, Current Medical Literature, 19-20.

[4] Critical Care Medicine Branch of the Chinese Medical Association (2007) Diagnosis and treatment guidelines of patients with severe invasive fungal infections. Chinese Journal of Internal Medicine, 46, 960-966.

[5] Dong, C.H., Wei, J., Zhi, H., et al. (2005) Infection and resistance of fungus. Chinese Journal of Nosocomiology, 15, 345-347.

[6] Julie, P. and William, M.L. (2005) The management of acute liver failure. Hepatology, 41, 1179-1196. doi:10.1002/hep.20703

[7] Zhang, X.H., Zhang, G.H., Man, C.J., et al. (2004) Clinical study on the severe hepatitis with nosocomial fungal infections and risk factors. Chinese Journal of Hepatology, 12, 389-391.

[8] Min, X., Qiang, C. and Qian, C.F. (2007) Clinical analysis of 186 severe hepatitis and cirrhosis patients with nosocomial fungal infections. Journal of Clinical Hepatology, 10, 24-27.

[9] Qi, F.X., Mei, L., Bing, L., et al. (2004) Risk factors of fungal colonization in patients with chronic liver disease: a prospective study. Chinese Journal of Nosocomiology, 14, 1344-1346.

[10] Segal, B.H., Amyroudis, N.G., Battiwalla, M., et al. (2007) Prevention and early treatment of invasive fungal infection in patients with cancer and neutropenia and in stem cell transplant recipients in the era of newer broadspectrum antifungal agents and diagnostic adjuncts. Clinical Infective Disease, 44, 402-409. doi:10.1086/510677

[11] Rex, J.H. and Sobel, J.D. (2001) Prophylactic antifungal therapy in the intensive care unit. Clinical Infective Disease, 32, 1191-1200. doi:10.1086/319763

[12] Pfaller, M.A., Jones, R.N., Messer, S.A., et al. (1998) National surveillance of nosocomial blood stream infection due to Candida albicans: Frequency of occurrence and antifungal susceptibility in the SCOPE Program. Diagnostic Microbiology and Infectious Disease, 31, 327-332. doi:10.1016/S0732-8893(97)00240-X

[13] Vehreschild, J.J., Bohme, A., Reichert, D., et al. (2008) Treatment of invasive fungal infections in clinical practice: A multi-centre survey on customary dosing, treatment indications, efficacy and safety of voriconazole. International Journal of Hematology, 87, 126-131. doi:10.1007/s12185-008-0045-z

[14] Ying, J.W. (2008) Liver failure: Definitions, diagnosis and treatment. Journal of Clinical Hepatology, 16, 725727.

[15] Ju, P.D. and Chun, H.L., et al. (2007) Invasive diagnosis and treatment of fungal infections. Chinese Journal of Infection Control, 6, 359-364. 